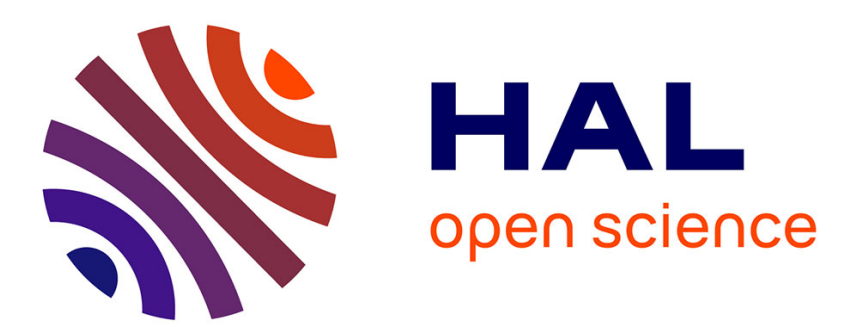

\title{
Stabilisation d'un actionneur électrohydraulique en considérant les dynamiques des distributeurs
}

\author{
Rachid Outbib, Hugues Rafaralahy, Mohamed Tafraouti
}

\section{To cite this version:}

Rachid Outbib, Hugues Rafaralahy, Mohamed Tafraouti. Stabilisation d'un actionneur électrohydraulique en considérant les dynamiques des distributeurs. Journal Européen des Systèmes Automatisés (JESA), 2005, 39 (9-10), pp.1025-1050. hal-00121739

\section{HAL Id: hal-00121739 \\ https://hal.science/hal-00121739}

Submitted on 21 Dec 2006

HAL is a multi-disciplinary open access archive for the deposit and dissemination of scientific research documents, whether they are published or not. The documents may come from teaching and research institutions in France or abroad, or from public or private research centers.
L'archive ouverte pluridisciplinaire HAL, est destinée au dépôt et à la diffusion de documents scientifiques de niveau recherche, publiés ou non, émanant des établissements d'enseignement et de recherche français ou étrangers, des laboratoires publics ou privés. 


\title{
Stabilisation d'un actionneur électrohydraulique en considérant les dynamiques des distributeurs
}

\author{
Rachid Outbib* - Hugues Rafaralahy $^{* *}$ - Mohamed Tafraouti** \\ ${ }^{*}$ L2ES \\ Université de Technologie de Belfort-Montbéliard \\ 90010 Belfort \\ rachid.outbib@utbm.fr \\ ${ }^{* *}$ CRAN, UMR 7039, Université Henri Poincaré - Nancy I, I.U.T. de Longwy \\ 186 rue de Lorraine \\ 54400 Cosnes-et-Romain \\ Hugues.Rafaralahy@iut-longwy.uhp-nancy.fr
}

\begin{abstract}
RESUME - Cet article est dédié à la stabilisation d'un actionneur électrohydraulique en tenant compte, dans la synthèse de la loi de commande, de la dynamique des distributeurs. Le comportement des actionneurs à fluide sous pression peuvent êtres décrits par des équations différentielles non linéaires qui sont seulement continues (c'est-à-dire non dérivables). Nous proposons dans cet article une extension de certains résultats de la littérature sur la stabilisation d'une classe de systèmes non linéaires lorsque le champ de vecteurs définissant le système est seulement continu. Il est à noter que la loi de commande proposée est donnée explicitement et elle est appliquée à la stabilisation d'un vérin hydraulique en tenant compte de la dynamique des distributeurs et ceci sans effectuer aucune linéarisation du modèle.

ABSTRACT - This paper is devoted to the stabilization of an electrohydraulic actuator taking into account the dynamics of the valves. The behaviour of actuators in fluid power systems can be described by non linear differential equations which are only continuous (i.e. not of class $C^{l}$ ). We propose in this paper an extension of some results on the stabilization of a class of non linear systems when the vector fields are only continuous. Notice that the obtained control law is given explicitely and is applied to the stabilization of an hydraulic cylinder taking into account the dynamics of the valves and without any kind of linearization.
\end{abstract}

MOTS-CLES: Stabilisation par retour d'état ; Actionneur électrohydraulique ; Systèmes non linéaires.

KEYWORDS: Feedback stabilization ; Electrohydraulic actuator ; Non linear systems. 


\section{Introduction}

La technologie hydraulique est largement utilisée dans l'industrie. Plus précisément, les actionneurs électrohydrauliques sont souvent sollicités dès lors que les efforts mis en jeu sont importants. Pour décrire l'évolution des processus électrohydrauliques, et compte tenu de la complexité de leur nature, les modèles utilisés sont en général non linéaires. Cependant, et concernant l'asservissement de ce type de procédé, les méthodes classiques dans la littérature sont celles qui sont fondées sur les techniques de linéarisation (voir (Merritt, 1967), (Cloy et Martin, 1980), (Watton, 1989), (Piché et al., 1992) et (Hahn et al., 1994)). Cette approche peut s'avérer tout à fait pertinente. En effet, elle a permis de répondre à beaucoup de problèmes et a conduit à de bons résultats expérimentaux (voir par exemple (Yao et al., 2000)). Néanmoins, elle peut présenter quelques inconvénients. Par exemple, une linéarisation autour d'un point de fonctionnement revêt un caractère local et donc cette technique devient inappropriée si l'on cherche à opérer sur une large plage de fonctionnement.

Cet article traite du problème de la commande des processus électrohydrauliques par une approche non linéaire. Plus précisément, le but principal est de proposer une nouvelle stratégie pour la stabilisation d'actionneurs électrohydrauliques en considérant la dynamique des distributeurs ${ }^{1}$.

Actuellement, dans la littérature, certains travaux ont été consacrés au problème de la stabilisation des systèmes électrohydrauliques par des techniques non linéaires (voir par exemple (Richard et Outbib, 1995), (Richard et al., 1997), et (Khorrami et Jain, 1998)). Cependant, les résultats établis concernent essentiellement le comportement de l'actionneur électrohydraulique et ne prennent pas en compte la dynamique des distributeurs. Concernant la commande des processus électrohydrauliques prenant en compte la dynamique des distributeurs, des travaux de recherche ont été dédiés à ce sujet (voir par exemple (Kim et Tsao, 2000)). Néanmoins, les résultats proposés reposent en général sur des techniques de linéarisation.

Un modèle décrivant l'évolution du processus électrohydraulique fondé sur une première approximation des dynamiques des distributeurs, et suite à un retour d'état préliminaire, peut être exprimé sous la forme

$$
\left\{\begin{array}{l}
\dot{x}=f(x, \chi) \\
\dot{\chi}=u
\end{array}\right.
$$

où $\mathrm{x}$ désigne l'état de l'actionneur, $\chi$ celui des distributeurs et $\mathrm{u}$ est la commande.

D'une façon générale, et indépendamment des modèles issus de l'électrohydraulique, la stabilisation du système non linéaire [1], où $(\mathrm{x}, \chi) \in \mathfrak{R}^{\mathrm{n}} \times \Re^{\mathrm{m}}$ désigne l'état du système et $\mathrm{u} \in \mathfrak{R}^{\mathrm{m}}$ représente la commande, $\mathrm{a}$ suscité l'intérêt de plusieurs auteurs (voir par exemple (Byrnes et Isidori, 1989), (Kokotovic et Sussmann, 1989), (Coron et Praly, 1991), (Outbib et Sallet, 1994), (Outbib et Jghima, 1998) et (Outbib et Aggoune, 1999)). Pour stabiliser le système [1], une méthode classique consiste d'abord à stabiliser le système réduit

$$
\dot{x}=f(x, u)
$$

puis de déduire un retour d'état stabilisant pour le système [1] à partir de celui de [2]. A l'origine, ce résultat a été établi dans le cas où $f$ est au moins de classe $C^{1}$. Le principe de la démonstration repose sur la régularité de $\mathrm{f}$. Plus précisément, et comme f est dérivable, il est possible d'effectuer la décomposition suivante :

$$
f(x, \chi)=f_{0}(x)+F(x, \chi) \chi
$$

\footnotetext{
${ }^{1}$ Tout le long de cet article, on utilisera la terminologie « distributeur » pour désigner indifféremment une servovalve ou un distributeur proportionnel.
} 
où $f_{0}(x) \equiv f(x, 0)$ et $F$ une fonction continue. Ainsi, et pour s'affranchir de l'hypothèse de dérivabilité de $f$, on peut supposer que $\mathrm{f}$ est seulement continue mais qu'elle vérifie l'hypothèse $[\mathrm{H}]$ (voir par exemple ((Freeman et Kokotovic, 1996), page 108)). Depuis, plusieurs généralisations de ce résultat ont été proposées. On peut citer, par exemple, les deux améliorations suivantes. La première (cf. (Tanner et Kyriakopoulos, 2003), Théorème 1), et sous l'hypothèse $[\mathrm{H}]$, concerne le cas où $\mathrm{f}_{0}$ et $\mathrm{F}$ sont des fonctions discontinues. La deuxième (cf. (Outbib et Aggoune, 1999)) traite du cas où l'hypothèse [H] n'est pas vérifiée et s'intéresse à la stabilisation du système [1] par retour d'état continu. Un exemple simple est le cas suivant

$$
\mathrm{f}(\mathrm{x}, \chi)=-\mathrm{x}+\chi^{1 / 3} \text { où }(\mathrm{x}, \chi) \in \mathfrak{R}^{2} .
$$

L'objectif de cet article est double. D'une part, nous allons donner un résultat sur la stabilisation du système [1] lorsque f est seulement continue. Il s'agit d'une amélioration du résultat proposé dans (Outbib et Aggoune, 1999). D'autre part, nous allons proposer une nouvelle stratégie, fondée sur ce nouveau résultat, pour synthétiser un retour d'état stabilisant pour les systèmes électrohydrauliques en tenant compte des dynamiques des distributeurs et des non-linéarités naturelles du système, et ceci sans effectuer aucune linéarisation.

Cet article est organisé de la façon suivante. Dans le deuxième paragraphe, une description du processus électrohydraulique considéré est donnée. Le troisième paragraphe est consacré à la méthode de stabilisation. Le quatrième paragraphe contient le résultat principal sur la stabilisation du processus électrohydraulique. Finalement, et pour illustrer les performances de la loi de commande proposée, des résultats de simulations sont donnés au cinquième paragraphe.

\section{Description du processus électrohydraulique considéré}

L'objectif de cette section est de présenter puis de décrire l'évolution du processus électrohydraulique considéré. Il s'agit d'un actionneur électrohydraulique constitué par un vérin double effet et une charge inertielle commandés par deux distributeurs. Dans la littérature, la modélisation de ce type de processus (voir (Blackburn et al., 1966), (Merritt, 1967), (Cloy et Martin, 1980), (Lebrun, 1986), (Watton, 1989) et (Maré, 1993)) repose essentiellement sur les lois d'évolution des pressions dans chaque chambre du vérin, les lois d'écoulement du fluide à travers les restrictions variables des distributeurs et les lois de la mécanique de l'ensemble mobile constitué par la charge inertielle et la tige du vérin (voir figure 1).

Les dynamiques d'évolution des pressions sont obtenues en effectuant le bilan massique dans chaque chambre du vérin, considérée comme un volume variable, soit

$$
\frac{\mathrm{dP}}{\mathrm{dt}}=\frac{\beta_{\mathrm{e}}}{\mathrm{V}}\left(\sum_{\mathrm{i}} \overline{\mathrm{Q}}_{\mathrm{e}-\mathrm{i}}-\sum_{\mathrm{j}} \overline{\mathrm{Q}}_{\mathrm{s}-\mathrm{j}}-\frac{\mathrm{dV}}{\mathrm{dt}}\right)
$$

où $\bar{Q}_{e-i}$ et $\bar{Q}_{s-j}$ représentent respectivement les débits volumiques entrants et sortants, $\beta_{\mathrm{e}}$ est le module de compressibilité effective (tenant compte de la compressibilité de l'huile, de l'effet de l'air entrâné et de l'élasticité de l'enveloppe du volume considéré), $\mathrm{V}$ désigne le volume variable considéré et $\mathrm{P}$ dénote la pression du fluide dans ce volume.

En appliquant la relation [3] à chaque chambre du vérin, nous obtenons

$$
\left\{\begin{array}{l}
\frac{\mathrm{dP}_{1}}{\mathrm{dt}}=\frac{\beta_{\mathrm{e}}}{\mathrm{V}_{0}+\mathrm{S}_{1} \mathrm{y}}\left(\overline{\mathrm{Q}}_{1}\left(\overline{\mathrm{u}}_{1}, \mathrm{P}_{1}\right)-\mathrm{S}_{1} \mathrm{v}\right) \\
\frac{\mathrm{dP}_{2}}{\mathrm{dt}}=\frac{\beta_{\mathrm{e}}}{\mathrm{V}_{0}-\mathrm{S}_{2} \mathrm{y}}\left(\overline{\mathrm{Q}}_{2}\left(\overline{\mathrm{u}}_{2}, \mathrm{P}_{2}\right)+\mathrm{S}_{2} \mathrm{v}\right)
\end{array}\right.
$$

$\mathrm{y}$ et $\mathrm{v}$ représentent respectivement le déplacement et la vitesse de la tige du vérin et $\mathrm{V}_{0}$ est le demi-volume du vérin défini par 


$$
\mathrm{V}_{0}=\frac{\mathrm{S}_{1} \mathrm{~S}_{2}}{\mathrm{~S}_{1}+\mathrm{S}_{2}} \ell+\mathrm{V}_{\mathrm{m}}
$$

où $S_{1}$ et $S_{2}$ sont les sections utiles et $\ell$ et $V_{m}$ désignent respectivement la course du vérin et le volume mort.

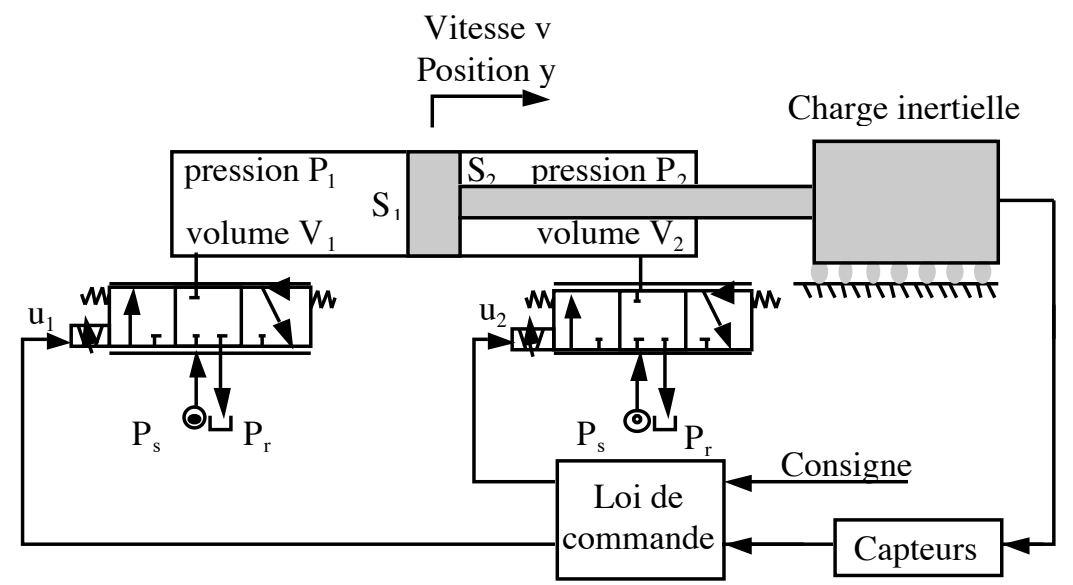

Figure 1. Représentation schématique de l'actionneur électrohydraulique.

Les expressions des lois de débit $\overline{\mathrm{Q}}_{1}$ et $\overline{\mathrm{Q}}_{2}$ sont obtenues en supposant que les écoulements à travers les restrictions des distributeurs sont en régime turbulent et à découvrement nul (voir par exemple (Merritt, 1967))

$$
\begin{gathered}
\overline{\mathrm{Q}}_{1}\left(\overline{\mathrm{u}}_{1}, \mathrm{P}_{1}\right)=\left\{\begin{array}{lll}
\overline{\mathrm{k}}_{\mathrm{q} 1} \overline{\mathrm{u}}_{1} \sqrt{\left|\mathrm{P}_{\mathrm{s}}-\mathrm{P}_{1}\right|} \operatorname{sign}\left(\mathrm{P}_{\mathrm{s}}-\mathrm{P}_{1}\right) & \text { si } & \overline{\mathrm{u}}_{1} \geq 0 \\
\overline{\mathrm{k}}_{\mathrm{q} 1} \overline{\mathrm{u}}_{1} \sqrt{\left|\mathrm{P}_{1}-\mathrm{P}_{\mathrm{r}}\right|} \operatorname{sign}\left(\mathrm{P}_{1}-\mathrm{P}_{\mathrm{r}}\right) & \text { si } & \overline{\mathrm{u}}_{1}<0
\end{array}\right. \\
\overline{\mathrm{Q}}_{2}\left(\overline{\mathrm{u}}_{2}, \mathrm{P}_{2}\right)=\left\{\begin{array}{lll}
\overline{\mathrm{k}}_{\mathrm{q} 2} \overline{\mathrm{u}}_{2} \sqrt{\left|\mathrm{P}_{\mathrm{s}}-\mathrm{P}_{2}\right|} \operatorname{sign}\left(\mathrm{P}_{\mathrm{s}}-\mathrm{P}_{2}\right) & \text { si } & \overline{\mathrm{u}}_{2} \geq 0 \\
\overline{\mathrm{k}}_{\mathrm{q} 2} \overline{\mathrm{u}}_{2} \sqrt{\left|\mathrm{P}_{2}-\mathrm{P}_{\mathrm{r}}\right|} \operatorname{sign}\left(\mathrm{P}_{2}-\mathrm{P}_{\mathrm{r}}\right) & \text { si } & \overline{\mathrm{u}}_{2}<0
\end{array}\right.
\end{gathered}
$$

où $\mathrm{P}_{\mathrm{s}}$ est la pression d'alimentation, $\quad \mathrm{P}_{\mathrm{r}}$ désigne la pression de retour, $\quad \overline{\mathrm{u}}_{1}$ et $\overline{\mathrm{u}}_{2}$ représentent les variables électriques de commande (tensions ou courants) des distributeurs et $\overline{\mathrm{k}}_{\mathrm{qj}}(\mathrm{j}=1,2)$ des constantes strictement positives caractérisant les distributeurs

$$
\overline{\mathrm{k}}_{\mathrm{qj}}=\frac{\overline{\mathrm{Q}}_{\mathrm{nj}}}{\overline{\mathrm{u}}_{\mathrm{nj}} \sqrt{\Delta \mathrm{P}_{\mathrm{n}}}}
$$

$\overline{\mathrm{Q}}_{\mathrm{nj}}, \overline{\mathrm{u}}_{\mathrm{nj}}$ et $\Delta \mathrm{P}_{\mathrm{n}}$ désignent respectivement les valeurs nominales du débit, de la commande et de la perte de charge par arête active correspondant à la zone linéaire de la relation débit-courant du distributeur.

Les équations mécaniques sont obtenues en utilisant le principe de Newton à l'ensemble mobile constitué par la tige du vérin et la masse inertielle 


$$
\left\{\begin{array}{l}
\frac{\mathrm{dv}}{\mathrm{dt}}=\frac{1}{\mathrm{M}}\left(\mathrm{S}_{1} \mathrm{P}_{1}-\mathrm{S}_{2} \mathrm{P}_{2}\right) \\
\frac{\mathrm{dy}}{\mathrm{dt}}=\mathrm{v}
\end{array}\right.
$$

où $\mathrm{M}$ représente la masse de l'ensemble mobile, $\mathrm{v}$ la vitesse de déplacement du vérin et y sa position. Si l'on ne tient pas compte des dynamiques des distributeurs, le comportement de l'actionneur électrohydraulique est donc décrit par le modèle d'état non linéaire suivant :

$$
\left\{\begin{aligned}
\frac{\mathrm{dP}}{\mathrm{dt}} & =\frac{\beta_{\mathrm{e}}}{\mathrm{V}_{0}+\mathrm{S}_{1} \mathrm{y}}\left(\overline{\mathrm{Q}}_{1}\left(\overline{\mathrm{u}}_{1}, \mathrm{P}_{1}\right)-\mathrm{S}_{1} \mathrm{v}\right) \\
\frac{\mathrm{dP}}{\mathrm{dt}} & =\frac{\beta_{\mathrm{e}}}{\mathrm{V}_{0}-\mathrm{S}_{2} \mathrm{y}}\left(\overline{\mathrm{Q}}_{2}\left(\overline{\mathrm{u}}_{2}, \mathrm{P}_{2}\right)+\mathrm{S}_{2} \mathrm{v}\right) \\
\frac{\mathrm{dv}}{\mathrm{dt}} & =\frac{1}{\mathrm{M}}\left(\mathrm{S}_{1} \mathrm{P}_{1}-\mathrm{S}_{2} \mathrm{P}_{2}\right) \\
\frac{\mathrm{dy}}{\mathrm{dt}} & =\mathrm{v}
\end{aligned}\right.
$$

où les expressions des débits $\overline{\mathrm{Q}}_{1}$ et $\overline{\mathrm{Q}}_{2}$ sont données par les relations [5-a] et [5-b].

Dans la littérature sur la stabilisation des systèmes, des lois de commande non linéaires ont été proposées pour ce type de modèle (voir par exemple (Richard et Outbib, 1995) et (Richard et al., 1997)). En général, les lois de commande sont synthétisées sous l'hypothèse que les dynamiques des distributeurs sont suffisamment rapides en comparaison avec celle du vérin. En pratique, ce n'est pas toujours le cas, surtout lorsque l'on utilise des distributeurs proportionnels moins onéreux à la place des servovalves.

L'objectif principal de cet article est de proposer une nouvelle stratégie pour la stabilisation des systèmes électrohydrauliques prenant en considération les dynamiques des distributeurs. Plus précisément, nous supposons que les transferts entre les variables de commande $u_{1}$ et $u_{2}$ et les positions des tiroirs des distributeurs $\mathrm{y}_{\mathrm{s} 1}$ et $\mathrm{y}_{\mathrm{s} 2}$ sont régis par des équations différentielles linéaires du premier ordre (c.f. (Khorrami et Jain, 1998) et (Yao et al., 2000))

$$
\left\{\begin{array}{l}
\frac{\mathrm{dy}_{\mathrm{sl}}}{\mathrm{dt}}=-\frac{1}{\tau_{1}}\left(\mathrm{y}_{\mathrm{s} 1}+\mathrm{k}_{\mathrm{s} 1} \mathrm{u}_{1}\right) \\
\frac{\mathrm{dy} \mathrm{s}_{\mathrm{s} 2}}{\mathrm{dt}}=-\frac{1}{\tau_{2}}\left(\mathrm{y}_{\mathrm{s} 2}+\mathrm{k}_{\mathrm{s} 2} \mathrm{u}_{2}\right)
\end{array}\right.
$$

où $\tau_{\mathrm{i}}(\mathrm{i}=1,2)$ et $\mathrm{k}_{\mathrm{si}}(\mathrm{i}=1,2)$ représentent respectivement les constantes de temps des distributeurs et les gains statiques.

Pour parvenir à un modèle décrivant l'évolution du processus électrohydraulique en tenant compte des dynamiques des distributeurs, on intègre les équations données par [8] dans le système [7]. Nous obtenons donc le modèle complet suivant : 


$$
\left\{\begin{aligned}
\frac{\mathrm{dP}_{1}}{\mathrm{dt}} & =\frac{\beta_{\mathrm{e}}}{\mathrm{V}_{0}+\mathrm{S}_{1} \mathrm{y}}\left(\mathrm{Q}_{1}\left(\mathrm{y}_{\mathrm{sl}}, \mathrm{P}_{1}\right)-\mathrm{S}_{1} \mathrm{v}\right) \\
\frac{\mathrm{dP}}{\mathrm{dt}} & =\frac{\beta_{\mathrm{e}}}{\mathrm{V}_{0}-\mathrm{S}_{2} \mathrm{y}}\left(\mathrm{Q}_{2}\left(\mathrm{y}_{\mathrm{s} 2}, \mathrm{P}_{2}\right)+\mathrm{S}_{2} \mathrm{v}\right) \\
\frac{\mathrm{dv}}{\mathrm{dt}} & =\frac{1}{\mathrm{M}}\left(\mathrm{S}_{1} \mathrm{P}_{1}-\mathrm{S}_{2} \mathrm{P}_{2}\right) \\
\frac{\mathrm{dy}}{\mathrm{dt}} & =\mathrm{v} \\
\frac{\mathrm{dy} \mathrm{y}_{\mathrm{s}}}{\mathrm{dt}} & =-\frac{1}{\tau_{1}}\left(\mathrm{y}_{\mathrm{sl}}+\mathrm{k}_{\mathrm{sl}} \mathrm{u}_{1}\right) \\
\frac{\mathrm{dy} \mathrm{s} 2}{\mathrm{dt}} & =-\frac{1}{\tau_{2}}\left(\mathrm{y}_{\mathrm{s} 2}+\mathrm{k}_{\mathrm{s} 2} \mathrm{u}_{2}\right)
\end{aligned}\right.
$$

Les lois de débit $\mathrm{Q}_{1}\left(\mathrm{y}_{\mathrm{sl}}, \mathrm{P}_{1}\right)$ et $\mathrm{Q}_{2}\left(\mathrm{y}_{\mathrm{s} 2}, \mathrm{P}_{2}\right)$ sont données par

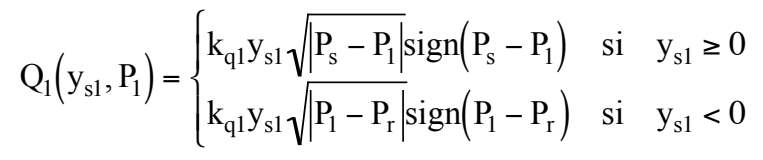

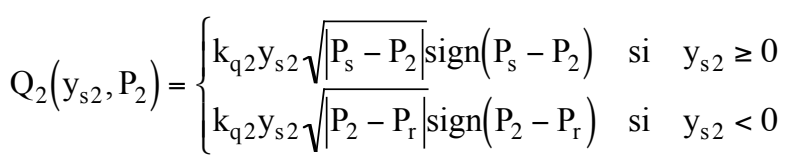

où $\mathrm{k}_{\mathrm{qj}}(\mathrm{j}=1$ ou 2 ) sont des constantes strictement positives vérifiant

$$
\mathrm{k}_{\mathrm{qj}}=\mathrm{k}_{\mathrm{sj}} \overline{\mathrm{k}}_{\mathrm{qj}} \cdot
$$

Le reste de cette section sera consacré à la définition des conditions d'équilibre et du domaine d'évolution de l'état du système électrohydraulique. Un raisonnement simple montre que les conditions d'équilibre du système [9] sont déterminées par les relations suivantes

$$
\begin{gathered}
P_{1}=P_{1}^{0}, P_{2}=P_{2}^{0}=\frac{S_{1}}{S_{2}} P_{1}^{0}, v=v^{0}=0, y=y^{0}, y_{s 1}=y_{s 1}^{0}=0, y_{s 2}=y_{s 2}^{0}=0, \\
\text { et } u_{1}=u_{1}^{0}=0, u_{2}=u_{2}^{0}=0
\end{gathered}
$$

Il convient de préciser que la pression $\mathrm{P}_{1}^{0}$ et la position $\mathrm{y}^{0}$ ne sont pas imposées par les conditions d'équilibre. Ainsi, un objectif de la loi de commande consistera à fixer les valeurs désirées de $\mathrm{P}_{1}^{0}$ et $\mathrm{y}^{0}$.

Par ailleurs, la loi de commande proposée doit respecter le domaine d'évolution de l'état du système, c'est-àdire tenir compte des contraintes physiques telles que la positivité des pressions, la course limitée de l'actionneur etc. Pour le système considéré, nous avons le domaine physique suivant :

$$
\begin{aligned}
\mathrm{D}= & \left\{(\mathrm{x}, \chi)=\left(\mathrm{P}_{1}, \mathrm{P}_{2}, \mathrm{v}, \mathrm{y}, \mathrm{y}_{\mathrm{s} 1}, \mathrm{y}_{\mathrm{s} 2}\right)^{\mathrm{T}} \in \mathfrak{R}^{6} /\right. \\
& \left.\mathrm{P}_{1}>0, \mathrm{P}_{2}>0, \mathrm{v} \in \Re,-\ell<\mathrm{y}<\ell,-\ell_{\mathrm{s} 1}<\mathrm{y}_{\mathrm{s} 1}<\ell_{\mathrm{s} 1},-\ell_{\mathrm{s} 2}<\mathrm{y}_{\mathrm{s} 2}<\ell_{\mathrm{s} 2}\right\}
\end{aligned}
$$




\section{Méthode de stabilisation}

Dans cette section, nous nous intéressons à la stabilisation par retour d'état continu du système non linéaire [1] quand la fonction $\mathrm{f}$ est seulement continue et où la condition $[\mathrm{H}]$ n'est pas forcément vérifiée. Plus précisément, il est bien connu que si le système [2] est stabilisable par une commande $\overline{\mathrm{u}}$ de classe $\mathrm{C}^{1}$, alors le système [1] est stabilisable par une loi de commande au moins continue si f est au moins de classe $\mathrm{C}^{1}$ où si elle est continue mais vérifie l'hypothèse $[\mathrm{H}]$. La démonstration de ce résultat est fondée essentiellement sur la décomposition donnée par $[\mathrm{H}]$.

Il est à noter que cette démonstration n'est plus valide si f est seulement continue mais ne vérifiant pas [H]. Il a été montré dans (Outbib et Aggoune, 1999) que ce résultat reste valide pour toute fonction $\mathrm{f}$ continue et même si l'hypothèse $[\mathrm{H}]$ n'est pas vérifiée. Cependant, ce résultat de stabilisation (voir (Outbib et Aggoune, 1999)) concerne le cas particulier où le retour d'état stabilisant $\bar{u}$ du système réduit [1] vérifie $\overline{\mathrm{u}} \equiv 0$. Dans ce qui suit, nous nous proposons de donner une extension de ce résultat dans le cas général même si $\overline{\mathrm{u}} \neq 0$.

En utilisant le Lemme 2 de (Outbib et Aggoune, 1999), nous pouvons écrire

$$
f(x, \chi)-f(x, \bar{u}(x))=F(x, \chi) H(\chi-\bar{u}(x))
$$

où $F$ et $H=\left(\begin{array}{c}h_{1} \\ \vdots \\ h_{m}\end{array}\right)$ sont des fonctions continues telles que :

(C1) $\quad \mathrm{h}_{\mathrm{i}}(\mathrm{z}) \mathrm{z}>0$ pour $\mathrm{z} \neq 0$,

(C2) et $\lim _{z \rightarrow+\infty}\left|h_{i}(z)\right|=+\infty$ pour $\mathrm{i}=1, \ldots, \mathrm{m}$.

En utilisant l'égalité ci-dessus, on peut réécrire le système [1] sous la forme

$$
\left\{\begin{array}{l}
\dot{\mathrm{x}}=\mathrm{f}(\mathrm{x}, \overline{\mathrm{u}}(\mathrm{x}))+\mathrm{F}(\mathrm{x}, \chi) \mathrm{H}(\chi-\overline{\mathrm{u}}(\mathrm{x})) \\
\dot{\chi}=\mathrm{v}
\end{array}\right.
$$

Il convient de préciser que l'existence de $\mathrm{F}$ et $\mathrm{H}$ est assurée par la continuité de $\mathrm{f}$ (voir lemme 2, (Outbib et Aggoune, 1999)).

Maintenant, nous allons énoncer et démontrer le résultat principal de cette section.

\section{Théorème 1}

Considérons le système [1] et supposons que f est au moins continue. Soit la commande $\overline{\mathrm{u}}$, de classe au moins $\mathrm{C}^{1}$ avec $\overline{\mathrm{u}}(0)=0$, telle que le système bouclé suivant :

$$
\dot{\mathrm{x}}=\mathrm{f}(\mathrm{x}, \overline{\mathrm{u}}(\mathrm{x}))
$$

est globalement asymptotiquement stable à l'origine. Supposons qu'il existe une fonction de Lyapunov $\mathrm{W}_{1}$, connue, telle que :

$$
\dot{\mathrm{W}}_{1}(\mathrm{x})=\left\langle\nabla \mathrm{W}_{1}(\mathrm{x}), \mathrm{f}(\mathrm{x}, \overline{\mathrm{u}}(\mathrm{x}))\right\rangle \leq 0 \text { pour tout } \mathrm{x} \in \mathfrak{R}^{\mathrm{n}},
$$

où $\nabla$ désigne le gradient. Alors, la loi de commande suivante 


$$
\mathrm{u}(\mathrm{x}, \chi)=\frac{\partial \overline{\mathrm{u}}}{\partial \mathrm{x}}(\mathrm{x}) \mathrm{f}(\mathrm{x}, \chi)-\nabla \mathrm{W}_{1}(\mathrm{x}) \mathrm{F}(\mathrm{x}, \chi)-\mathrm{k}(\chi-\overline{\mathrm{u}}(\mathrm{x}))
$$

où $\mathrm{k}$ est une constante strictement positive, rend l'origine du système [1] globalement asymptotiquement stable. $\square$

\section{Démonstration}

Soit $\mathrm{W}_{2}$ la fonction de Lyapunov définie par

$$
\mathrm{W}_{2}(\mathrm{x}, \chi)=\mathrm{W}_{1}(\mathrm{x})+\sum_{\mathrm{i}=1}^{\mathrm{m}}\left(\int_{0}^{x_{\mathrm{i}}-\overline{\mathrm{u}}_{\mathrm{i}}(\mathrm{x})} \mathrm{h}_{\mathrm{i}}(\tau) \mathrm{d} \tau\right)
$$

La dérivée de $\mathrm{W}_{2}$ le long des trajectoires du système [1'] est donnée par

$$
\begin{aligned}
\dot{\mathrm{W}}_{2}(\mathrm{x}, \chi)= & \left\langle\nabla \mathrm{W}_{1}(\mathrm{x}), \mathrm{f}(\mathrm{x}, \overline{\mathrm{u}}(\mathrm{x}))\right\rangle+\nabla \mathrm{W}_{1}(\mathrm{x}) \mathrm{F}(\mathrm{x}, \chi) \mathrm{H}(\chi-\overline{\mathrm{u}}(\mathrm{x}))- \\
& \left\langle\left(\frac{\partial \overline{\mathrm{u}}}{\partial \mathrm{x}}(\mathrm{x})\right)^{\mathrm{T}} \mathrm{H}(\chi-\overline{\mathrm{u}}(\mathrm{x})), \mathrm{f}(\mathrm{x}, \chi)\right\rangle+\langle\mathrm{H}(\chi-\overline{\mathrm{u}}(\mathrm{x})), \mathrm{u}\rangle \\
= & \left\langle\nabla \mathrm{W}_{1}(\mathrm{x}), \mathrm{f}(\mathrm{x}, \overline{\mathrm{u}}(\mathrm{x}))\right\rangle+ \\
& \left\langle\left(\nabla \mathrm{W}_{1}(\mathrm{x}) \mathrm{F}(\mathrm{x}, \chi)-\frac{\partial \overline{\mathrm{u}}}{\partial \mathrm{x}}(\mathrm{x}) \mathrm{f}(\mathrm{x}, \chi)+\mathrm{u}\right), \mathrm{H}(\chi-\overline{\mathrm{u}}(\mathrm{x}))\right\rangle .
\end{aligned}
$$

En considérant la loi de commande [13], nous obtenons

$$
\dot{\mathrm{W}}_{2}(\mathrm{x}, \chi)=\left\langle\nabla \mathrm{W}_{1}(\mathrm{x}), \mathrm{f}(\mathrm{x}, \overline{\mathrm{u}}(\mathrm{x}))\right\rangle-\mathrm{k}\langle(\chi-\overline{\mathrm{u}}(\mathrm{x})), \mathrm{H}(\chi-\overline{\mathrm{u}}(\mathrm{x}))\rangle .
$$

Maintenant, en utilisant l'inégalité [12] et en tenant compte des propriétés de la fonction $\mathrm{H}$, nous pouvons déduire que

$$
\dot{\mathrm{W}}_{2}(\mathrm{x}, \chi) \leq 0 \text { pour tout }(\mathrm{x}, \chi) \in \Re^{\mathrm{n}} \times \Re^{\mathrm{m}} .
$$

Le système [1], bouclé par la loi de commande [13], est donc stable à l'origine. Pour établir la stabilité asymptotique globale de l'origine, nous utilisons le principe d'invariance de La Salle (La Salle et Lefschetz, 1961).

Soit $\Omega$ l'ensemble défini par

$$
\Omega=\left\{(\mathrm{x}, \chi) \in \mathfrak{R}^{\mathrm{n}} \times \Re^{\mathrm{m}} / \dot{\mathrm{W}}_{2}(\mathrm{x}, \chi)=0\right\} .
$$

ou, de manière équivalente, 


$$
\Omega=\left\{(\mathrm{x}, \chi) \in \mathfrak{R}^{\mathrm{n}} \times \mathfrak{R}^{\mathrm{m}} /\left\langle\nabla \mathrm{W}_{1}(\mathrm{x}), \mathrm{f}(\mathrm{x}, \overline{\mathrm{u}}(\mathrm{x}))\right\rangle=0 \text { et } \chi=\overline{\mathrm{u}}(\mathrm{x})\right\} .
$$

Soit $\gamma(\mathrm{t})=\left[\gamma_{1}^{\mathrm{T}}(\mathrm{t}), \gamma_{2}^{\mathrm{T}}(\mathrm{t})\right]^{\mathrm{T}}$ une trajectoire du système [1], bouclé par la loi de commande [13], pour laquelle $\mathrm{W}_{2}$ est constante. Il est aisé de constater que $\gamma_{1}(\mathrm{t})$ est une trajectoire de [11] pour laquelle $\mathrm{W}_{1}$ est constante. Compte tenu de l'hypothèse que le système [11] est globalement asymptotiquement stable, nous avons $\gamma_{1}(t) \equiv 0$. Ce qui implique que $\dot{\gamma}_{1}(\mathrm{t}) \equiv 0$, et en utilisant la définition de $\Omega$ et le fait que $\overline{\mathrm{u}}(0)=0$ nous obtenons $\gamma_{2}(\mathrm{t}) \equiv 0$. Le plus grand ensemble invariant de $\Omega$ est donc réduit à l'origine.

Finalement, nous pouvons conclure que le système [1] bouclé par la loi de commande [13] est globalement asymptotiquement stable à l'origine.

REMARQUE. - Le théorème précédent, et pour des raisons de simplicité, a été énoncé dans le cas où le point de fonctionnement est l'origine. Il est à noter que ce résultat, modulo quelques modifications dans la démonstration, reste valable pour $\mathrm{x}^{0}$, un point d'équilibre autre que l'origine.

Remarque. - Comme la fonction F est utilisée pour synthétiser la loi de commande et qu'elle est assurée d'être continue mais pas forcément de Lipschitz, alors le Théorème classique sur l'unicité des solutions n'est pas applicable pour le système bouclé [1'][13].

\section{Stabilisation de l'actionneur électrohydraulique}

Avant de présenter notre stratégie pour la stabilisation du processus électrohydraulique en prenant en considération les dynamiques des distributeurs, nous allons d'abord rappeler un résultat sur la stabilisation du système [7], proposé dans (Richard et Outbib, 1995) et (Richard et al., 1997). Il s'agit du cas où la synthèse du retour d'état ne tient pas compte des dynamiques des distributeurs.

Le modèle d'état décrivant l'évolution du processus électrohydraulique [7], sans tenir compte des dynamiques des distributeurs, peut être écrit sous la forme suivante

$$
\dot{\mathrm{x}}(\mathrm{t})=\phi(\mathrm{x}(\mathrm{t}))+\sum_{\mathrm{i}=1}^{2} \mathrm{~g}_{\mathrm{i}}(\mathrm{x}(\mathrm{t})) \varphi_{\mathrm{i}}\left(\mathrm{x}(\mathrm{t}), \overline{\mathrm{u}}_{\mathrm{i}}\right) \overline{\mathrm{u}}_{\mathrm{i}}
$$

En effet, dans le cas du modèle [7], il suffit de poser

$$
x(t)=\left(\begin{array}{c}
P_{1}(t) \\
P_{2}(t) \\
v(t) \\
y(t)
\end{array}\right), \phi(x(t))=\left(\begin{array}{c}
\frac{-S_{1} \beta_{e}}{V_{0}+S_{1} y} \\
\frac{S_{2} \beta_{e}}{V_{0}-S_{2} y} \\
\frac{1}{M}\left(S_{1} P_{1}-S_{2} P_{2}\right) \\
v
\end{array}\right), g_{1}(x)=\left(\begin{array}{c}
\frac{\beta_{e}}{V_{0}+S_{1} y} \\
0 \\
0 \\
0
\end{array}\right), g_{2}(x)=\left(\begin{array}{c}
0 \\
\frac{\beta_{e}}{V_{0}-S_{2} y} \\
0 \\
0
\end{array}\right)
$$




$$
\text { et, pour i=1 ou 2, } \varphi_{\mathrm{i}}\left(\mathrm{x}, \overline{\mathrm{u}}_{\mathrm{i}}\right)= \begin{cases}\bar{\varphi}_{\mathrm{i}}(\mathrm{x})=\overline{\mathrm{k}}_{\mathrm{qi}} \sqrt{\left|\mathrm{P}_{\mathrm{s}}-\mathrm{P}_{\mathrm{i}}\right|} \operatorname{sign}\left(\mathrm{P}_{\mathrm{s}}-\mathrm{P}_{\mathrm{i}}\right) & \text { si } \overline{\mathrm{u}}_{\mathrm{i}} \geq 0 \\ \underline{\varphi}_{\mathrm{i}}(\mathrm{x})=\overline{\mathrm{k}}_{\mathrm{qi}} \sqrt{\mathrm{P}_{\mathrm{i}}-\mathrm{P}_{\mathrm{r}} \mid} \operatorname{sign}\left(\mathrm{P}_{\mathrm{i}}-\mathrm{P}_{\mathrm{r}}\right) & \text { si } \overline{\mathrm{u}}_{\mathrm{i}}<0\end{cases}
$$

Dans ce qui suit, et par abus de notation, on écrira indifféremment $\varphi_{\mathrm{i}}\left(\mathrm{x}, \overline{\mathrm{u}}_{\mathrm{i}}\right)$ ou $\varphi_{\mathrm{i}}\left(\mathrm{P}_{\mathrm{i}}, \overline{\mathrm{u}}_{\mathrm{i}}\right), \mathrm{i}=1$ ou 2. Par ailleurs, nous utiliserons les notations suivantes. Soit $f: \Re^{n} \rightarrow \Re^{n}$ un champ de vecteur régulier. $\mathrm{Si}$ $\mathrm{V}: \mathfrak{R}^{\mathrm{n}} \rightarrow \mathfrak{R}$ est une fonction différentiable, alors on définit sa dérivée le long de $\mathrm{f}$ par

$$
\mathrm{L}_{\mathrm{f}} \mathrm{V}=\sum_{\mathrm{i}=1}^{\mathrm{n}} \mathrm{f}_{\mathrm{i}} \frac{\partial \mathrm{V}}{\partial \mathrm{x}_{\mathrm{i}}}
$$

et par récurrence

$$
\mathrm{L}_{\mathrm{f}}^{0} \mathrm{~V}=\mathrm{V} \text { et } \mathrm{L}_{\mathrm{f}}^{\mathrm{k}} \mathrm{V}=\mathrm{L}_{\mathrm{f}}\left(\mathrm{L}_{\mathrm{f}}^{\mathrm{k}-1} \mathrm{~V}\right) \forall \mathrm{k} \geq 1
$$

Le Théorème 2 énonce un résultat sur la stabilisation des systèmes non linéaires dont la structure est celle décrite par [15], et ceci indépendamment des modèles issus de l'électrohydraulique.

Théorème 2 (Richard et al., 1997)

Supposons qu'il existe des fonctions continues $\mathrm{q}_{\mathrm{i}}: \Re \rightarrow \Re$ et $\mathrm{r}_{\mathrm{i}}: \Re \rightarrow \Re(\mathrm{i}=1$ ou 2$)$ vérifiant :

(C3) $\mathrm{q}_{\mathrm{i}}(\mathrm{z}) \mathrm{z}<0$ pour $\mathrm{z} \neq 0$ et $\mathrm{q}_{\mathrm{i}}(0)=0$,

(C4) $r_{i}(x)>0$.

S'il existe une fonction $\mathrm{W}_{1}$ vérifiant :

(C5) $\mathrm{W}_{1}(\mathrm{x})>0 \forall \mathrm{x} \neq \mathrm{x}^{0}$ et $\mathrm{W}_{1}\left(\mathrm{x}^{0}\right)=0$,

(C6) $\quad \mathrm{L}_{\phi} \mathrm{W}_{1}(\mathrm{x}) \leq 0$ et $\varphi_{\mathrm{i}}\left(\mathrm{x}, \mathrm{r}_{\mathrm{i}}(\mathrm{x}) \mathrm{q}_{\mathrm{i}}\left(\mathrm{L}_{\mathrm{g}_{\mathrm{i}}} \mathrm{W}_{1}(\mathrm{x})\right)\right)>0$,

(C7) et $\left\{\mathrm{x} / \mathrm{L}_{\phi}^{\mathrm{k}+1} \mathrm{~W}_{1}(\mathrm{x})=\mathrm{L}_{\phi}^{\mathrm{k}} \mathrm{L}_{\mathrm{g}_{\mathrm{i}}} \mathrm{V}(\mathrm{x})=0, \quad \forall \mathrm{k} \in \mathrm{N}, \mathrm{i}=1,2\right\}=\left\{\mathrm{x}^{0}\right\}$,

alors la loi de commande définie par

$$
\overline{\mathrm{u}}_{\mathrm{i}}(\mathrm{x})=\mathrm{r}_{\mathrm{i}}(\mathrm{x}) \mathrm{q}_{\mathrm{i}}\left(\mathrm{L}_{\mathrm{g}_{\mathrm{i}}} \mathrm{W}_{\mathrm{l}}(\mathrm{x})\right), \mathrm{i}=1 \text { ou } 2
$$

stabilise le système [15] au voisinage du point d'équilibre $\mathrm{x}^{0}$.

Démonstration : voir (Richard et al., 1997).

Notre stratégie de stabilisation des processus électrohydrauliques prenant en compte les dynamiques des distributeurs (voir modèle [9]) est composée de deux phases. Dans la première phase, et en se basant sur le résultat du Théorème 2, on calcule un retour d'état (sous la forme [17]) qui stabilise le système [7]. Puis, dans une deuxième phase, on synthétise une loi de commande qui stabilise le modèle [9]. Pour ce faire, on utilise le résultat du Théorème 1 et le retour d'état obtenu dans la première phase. Il est à noter que la connaissance d'une fonction de Lyapunov vérifiant les conditions (C5) et (C7) est nécessaire étant donnée que la première phase repose sur le résultat du Théorème 2. La construction de cette fonction de Lyapunov, désignée par $\mathrm{W}_{1}$ dans 
l'énoncé du Théorème 2, peut être réalisée en mettant en évidence des intégrales premières naturelles du système non commandé et en prenant en compte des considérations énergétiques. En effet, un raisonnement simple montre que l'énergie du système s'écrit sous la forme

$$
\mathrm{E}(\mathrm{x})=\frac{1}{2} \mathrm{Mv}^{2}+\beta_{\mathrm{e}}\left(\mathrm{V}_{0}+\mathrm{S}_{1} \mathrm{y}\right) \gamma\left(\mathrm{P}_{1}-\mathrm{P}_{1}^{0}\right)+\beta_{\mathrm{e}}\left(\mathrm{V}_{0}-\mathrm{S}_{2} \mathrm{y}\right) \gamma\left(\mathrm{P}_{2}-\mathrm{P}_{2}^{0}\right)
$$

où $\gamma$ est la fonction scalaire définie par

$$
\gamma(\mathrm{s})=\exp \left(\frac{\mathrm{s}}{\beta_{\mathrm{e}}}\right)-\frac{\mathrm{s}}{\beta_{\mathrm{e}}}-1
$$

Maintenant, pour construire une fonction de Lyapunov $\mathrm{W}_{1}$, à partir de la quantité $\mathrm{E}$ donnée par [18], de manière à ce qu'elle soit définie positive au point de fonctionnement $\mathrm{x}^{0}=\left(\mathrm{P}_{1}^{0}, \mathrm{P}_{2}^{0}, 0, \mathrm{y}^{0}\right)$ (point d'équilibre désiré), il suffit de rajouter R, une quantité positive choisie convenablement, soit

$$
\mathrm{W}_{1}(\mathrm{x})=\mathrm{E}(\mathrm{x})+\mathrm{R}(\mathrm{x})
$$

Un choix possible pour la fonction R (voir par exemple (Richard et Outbib, 1995)) est donné par

$$
\mathrm{R}(\mathrm{x})=\frac{\mathrm{b}}{2}\left\{\left(\left(\mathrm{~V}_{0}+\mathrm{S}_{1} \mathrm{y}\right) \exp \left(\frac{\mathrm{P}_{1}-\mathrm{P}_{1}^{0}}{\beta_{\mathrm{e}}}\right)-\mathrm{V}_{0}\right)^{2}+\left(\left(\mathrm{V}_{0}-\mathrm{S}_{2} \mathrm{y}\right) \exp \left(\frac{\mathrm{P}_{2}-\mathrm{P}_{2}^{0}}{\beta_{\mathrm{e}}}\right)-\mathrm{V}_{0}\right)^{2}\right\}
$$

où $\mathrm{b}$ est un réel positif.

Finalement, et en utilisant la fonction $\mathrm{W}_{1}$ définie par [19], un retour d'état stabilisant pour le système [7] est donné par

$$
\left\{\begin{array}{l}
\overline{\mathrm{u}}_{1}=-\mathrm{r}_{1}\left(\frac{\mathrm{L}_{\mathrm{g}_{1}} \mathrm{~W}_{1}(\mathrm{x})}{\mathrm{S}_{1}}\right)^{1+\frac{2}{\mathrm{p}}} \\
\overline{\mathrm{u}}_{2}=-\mathrm{r}_{2}\left(\frac{\mathrm{L}_{\mathrm{g}_{2}} \mathrm{~W}_{1}(\mathrm{x})}{\mathrm{S}_{2}}\right)^{1+\frac{2}{\mathrm{p}}}
\end{array}\right.
$$

où $\mathrm{p}$ est un entier impair et $\mathrm{r}_{\mathrm{i}}(\mathrm{i}=1,2)$ sont des réels positifs. Il convient de préciser que le paramètre $\mathrm{b}$, utilisé dans la relation [20] donc dans la définition de $\mathrm{W}_{1}$, doit être choisi suffisamment petit de telle sorte que la condition (C6) soit satisfaite (voir (Richard et al., 1997)).

La suite de cette section est consacrée à la synthèse d'un retour d'état stabilisant pour le système [9]. Un raisonnement simple montre que le modèle d'état [9] décrivant l'évolution du processus électrohydraulique, et suite à un retour d'état préliminaire ${ }^{2}$, peut être écrit sous la forme suivante :

2 On pose $\left\{\begin{array}{l}\mathrm{u}_{1}:=-\frac{1}{\tau_{1}}\left(\mathrm{y}_{\mathrm{s} 1}+\mathrm{k}_{\mathrm{s} 1} \mathrm{u}_{1}\right) \\ \mathrm{u}_{2}:=-\frac{1}{\tau_{2}}\left(\mathrm{y}_{\mathrm{s} 2}+\mathrm{k}_{\mathrm{s} 2} \mathrm{u}_{2}\right)\end{array}\right.$. 


$$
\left\{\begin{array}{l}
\dot{x}=f(x, \chi) \\
\dot{\chi}=u
\end{array}\right.
$$

avec $x=\left(\begin{array}{c}\mathrm{P}_{1} \\ \mathrm{P}_{2} \\ \mathrm{v} \\ \mathrm{y}\end{array}\right), \chi=\left(\begin{array}{c}\mathrm{y}_{\mathrm{s} 1} \\ \mathrm{y}_{\mathrm{s} 2}\end{array}\right)$ et $\mathrm{u}=\left(\begin{array}{l}\mathrm{u}_{1} \\ \mathrm{u}_{2}\end{array}\right)$ et $\mathrm{f}$ est le champ de vecteurs définissant le système, soit

$$
f(x, \chi)=\left(\begin{array}{c}
\frac{\beta_{e}}{V_{0}+S_{1} y}\left(Q_{1}\left(y_{s 1}, P_{1}\right)-S_{1} v\right) \\
\frac{\beta_{e}}{V_{0}-S_{2} y}\left(Q_{2}\left(y_{s 2}, P_{2}\right)+S_{2} v\right) \\
\frac{1}{M}\left(S_{1} P_{1}-S_{2} P_{2}\right) \\
v
\end{array}\right)
$$

Il est facile de voir que

$$
\begin{aligned}
\mathrm{f}(\mathrm{x}, \chi)-\mathrm{f}(\mathrm{x}, \overline{\mathrm{u}}) & =\left[\begin{array}{c}
\beta_{\mathrm{e}} \frac{\varphi_{1}\left(\mathrm{P}_{1}, \mathrm{y}_{\mathrm{s} 1}\right) \mathrm{y}_{\mathrm{s} 1}-\varphi_{1}\left(\mathrm{P}_{1}, \overline{\mathrm{u}}_{1}\right) \overline{\mathrm{u}}_{1}}{\mathrm{~V}_{0}+\mathrm{S}_{1} \mathrm{y}} \\
\beta_{\mathrm{e}} \frac{\varphi_{2}\left(\mathrm{P}_{2},-\overline{\mathrm{u}}_{2}\right) \overline{\mathrm{u}}_{2}-\varphi_{2}\left(\mathrm{P}_{2},-\mathrm{y}_{\mathrm{s} 2}\right) \mathrm{y}_{\mathrm{s} 2}}{\mathrm{~V}_{0}-\mathrm{S}_{2} \mathrm{y}} \\
0 \\
0
\end{array}\right] \\
& =\mathrm{F}(\mathrm{x}, \chi) \mathrm{H}(\chi-\overline{\mathrm{u}}(\mathrm{x}))=\underbrace{\left[\begin{array}{cc}
\mathrm{F}_{1} & 0 \\
0 & \mathrm{~F}_{2} \\
0 & 0 \\
0 & 0
\end{array}\right]}_{\mathrm{F}} \underbrace{\left[\begin{array}{c}
\left(\mathrm{y}_{\mathrm{s} 2}-\overline{\mathrm{u}}_{2}\right)^{\frac{1}{3}} \\
\left(\mathrm{y}_{\mathrm{s} 1}-\overline{\mathrm{u}}_{1}\right)^{\frac{1}{3}} \\
\mathrm{~s}^{3}
\end{array}\right.}_{\mathrm{H}(\chi-\overline{\mathrm{u}})}
\end{aligned}
$$

où $\mathrm{F}_{1}$ et $\mathrm{F}_{2}$ sont définies par

$$
\begin{gathered}
\mathrm{F}_{1}=\left\{\begin{array}{cc}
0 & \text { si } \mathrm{y}_{\mathrm{s} 1}=\overline{\mathrm{u}}_{1} \\
\beta_{\mathrm{e}} \frac{\varphi_{1}\left(\mathrm{P}_{1}, \mathrm{y}_{\mathrm{s} 1}\right) \mathrm{y}_{\mathrm{s} 1}-\varphi_{1}\left(\mathrm{P}_{1}, \overline{\mathrm{u}}_{1}\right) \overline{\mathrm{u}}_{1}}{\left(\mathrm{~V}_{0}+\mathrm{S}_{1} \mathrm{y}\right)\left(\mathrm{y}_{\mathrm{s} 1}-\overline{\mathrm{u}}_{1}\right)^{\frac{1}{3}}} & \text { sinon } \\
0 & \text { si } \mathrm{y}_{\mathrm{s} 2}=\overline{\mathrm{u}}_{2}
\end{array}\right. \\
\mathrm{F}_{2}=\left\{\begin{array}{cc}
\beta_{\mathrm{e}} \frac{\varphi_{2}\left(\mathrm{P}_{2},-\overline{\mathrm{u}}_{2}\right) \overline{\mathrm{u}}_{2}-\varphi_{2}\left(\mathrm{P}_{2},-\mathrm{y}_{\mathrm{s} 2}\right) \mathrm{y}_{\mathrm{s} 2}}{\left(\mathrm{~V}_{0}-\mathrm{S}_{2} \mathrm{y}\right)\left(\mathrm{y}_{\mathrm{s} 2}-\overline{\mathrm{u}}_{2}\right)^{\frac{1}{3}}} & \text { sinon }
\end{array}\right.
\end{gathered}
$$

Pour pouvoir utiliser le résultat du Théorème 1 , il reste à montrer que $F_{1}$ et $F_{2}$ sont bien des fonctions continues. Pour ce faire, on distingue deux cas. Tout d'abord, supposons que $\mathrm{y}_{\mathrm{sl}} \neq \overline{\mathrm{u}}_{1}$, alors il est facile de montrer que $\mathrm{F}_{1}$ est bien définie et est continue. Considérons maintenant le cas où $\mathrm{y}_{\mathrm{sl}}=\overline{\mathrm{u}}_{1}$. Il s'agit de montrer que pour $\lambda \in \Re$, nous avons 


$$
\lim _{\substack{\mathrm{y}_{\mathrm{s}}, \mathrm{u}_{1} \rightarrow \lambda \\ \mathrm{y}_{\mathrm{s} 1} \neq \mathrm{u}_{1}}} \beta_{\mathrm{e}} \frac{\varphi_{1}\left(\mathrm{P}_{1}, \mathrm{y}_{\mathrm{s} 1}\right) \mathrm{y}_{\mathrm{s} 1}-\varphi_{1}\left(\mathrm{P}_{1}, \overline{\mathrm{u}}_{1}\right) \overline{\mathrm{u}}_{1}}{\left(\mathrm{y}_{\mathrm{s} 1}-\overline{\mathrm{u}}_{1}\right)^{\frac{1}{3}}}=0
$$

Ce qui prouvera la continuité de $F_{1}$. Supposons que $\lambda \neq 0$. Alors pour $\left|\mathrm{y}_{\mathrm{s} 1}-\lambda\right|$ et $\left|\overline{\mathrm{u}}_{1}-\lambda\right|$ suffisamment petits, $\mathrm{y}_{\mathrm{s} 1}$ et $\overline{\mathrm{u}}_{1}$ sont de même signe, et donc $\varphi_{1}\left(\mathrm{P}_{1}, \mathrm{y}_{\mathrm{s} 1}\right)=\varphi_{1}\left(\mathrm{P}_{1}, \overline{\mathrm{u}}_{1}\right)$.

On en déduit que

$$
\beta_{\mathrm{e}} \frac{\varphi_{1}\left(\mathrm{P}_{1}, \mathrm{y}_{\mathrm{s} 1}\right) \mathrm{y}_{\mathrm{s} 1}-\varphi_{1}\left(\mathrm{P}_{1}, \overline{\mathrm{u}}_{1}\right) \overline{\mathrm{u}}_{1}}{\left(\mathrm{y}_{\mathrm{s} 1}-\overline{\mathrm{u}}_{1}\right)^{\frac{1}{3}}}=\varphi_{1}\left(\mathrm{P}_{1}, \mathrm{y}_{\mathrm{s} 1}\right)\left(\mathrm{y}_{\mathrm{s} 1}-\overline{\mathrm{u}}_{1}\right)^{\frac{2}{3}}
$$

ainsi la relation [24] est vérifiée.

Considérons maintenant le cas $\lambda=0$. Si $\mathrm{y}_{\mathrm{s} 1}$ et $\overline{\mathrm{u}}_{1}$ sont de même signe, alors il est facile de voir, en utilisant un raisonnement analogue à celui du cas $\lambda \neq 0$, que la relation [24] est vérifiée. Dans le cas où $\mathrm{y}_{\mathrm{s} 1}$ et $\overline{\mathrm{u}}_{1}$ sont de signes contraires, on peut utiliser la majoration suivante

$$
\left|\frac{\varphi_{1}\left(\mathrm{P}_{1}, \mathrm{y}_{\mathrm{s} 1}\right) \mathrm{y}_{\mathrm{s} 1}-\varphi_{1}\left(\mathrm{P}_{1}, \overline{\mathrm{u}}_{1}\right) \overline{\mathrm{u}}_{1}}{\left(\mathrm{y}_{\mathrm{s} 1}-\overline{\mathrm{u}}_{1}\right)^{\frac{1}{3}}}\right|<\operatorname{Sup}\left(\bar{\varphi}_{1}\left(\mathrm{P}_{1}\right), \underline{\varphi}_{1}\left(\mathrm{P}_{1}\right)\right) \times\left(\left|\mathrm{y}_{\mathrm{s} 1}\right|+\left|\overline{\mathrm{u}}_{1}\right|\right)^{\frac{2}{3}} .
$$

Alors, et comme

$$
\lim _{\substack{\mathrm{y}_{\mathrm{s}}, \overline{\mathrm{u}}_{\mathrm{u}} \rightarrow 0 \\ \mathrm{y}_{\mathrm{s} 1} \neq \overline{\mathrm{u}}_{1}}} \operatorname{Sup}\left(\bar{\varphi}_{1}\left(\mathrm{P}_{1}\right), \underline{\varphi}_{1}\left(\mathrm{P}_{1}\right)\right) \times\left(\left|\mathrm{y}_{\mathrm{s} 1}\right|+\left|\overline{\mathrm{u}}_{1}\right|\right)^{\frac{2}{3}}=0
$$

nous pouvons déduire que $F_{1}$ est continue au point $\left(\mathrm{y}_{\mathrm{s} 1}, \overline{\mathrm{u}}_{1}\right)=(0,0)$. Ceci termine la démonstration que $\mathrm{F} \quad$ 1 est continue.

D'une façon analogue, on peut montrer que $\mathrm{F}_{2}$ est continue.

Nous pouvons donc conclure qu'un retour d'état stabilisant pour le système [9] au voisinage du point de fonctionnement $\left(\mathrm{x}^{0^{\mathrm{T}}}, \chi^{0^{\mathrm{T}}}\right)=\left(\mathrm{P}_{1}^{0}, \mathrm{P}_{2}^{0}, 0, \mathrm{y}^{0}, 0,0\right)$ est donné par [13] où $\overline{\mathrm{u}}, \mathrm{W}_{1}$ et $\mathrm{F}$ sont définies respectivement par [21], [19] et [23]. Par ailleurs, une fonction de Lyapunov pour le système bouclé est la suivante

$$
\mathrm{W}_{2}(\mathrm{x})=\mathrm{W}_{1}(\mathrm{x})+\frac{3}{4}\left(\left(\mathrm{y}_{\mathrm{sl}}-\overline{\mathrm{u}}_{1}\right)^{\frac{4}{3}}+\left(\mathrm{y}_{\mathrm{s} 2}-\overline{\mathrm{u}}_{2}\right)^{\frac{4}{3}}\right)
$$

où $\mathrm{W}_{1}$ est donnée par [19]. 


\section{Simulations numériques}

Dans cette section, et pour illustrer les performances de la loi de commande proposée dans cet article (désignée dans la suite par commande I), des résultats de simulation sont présentés.

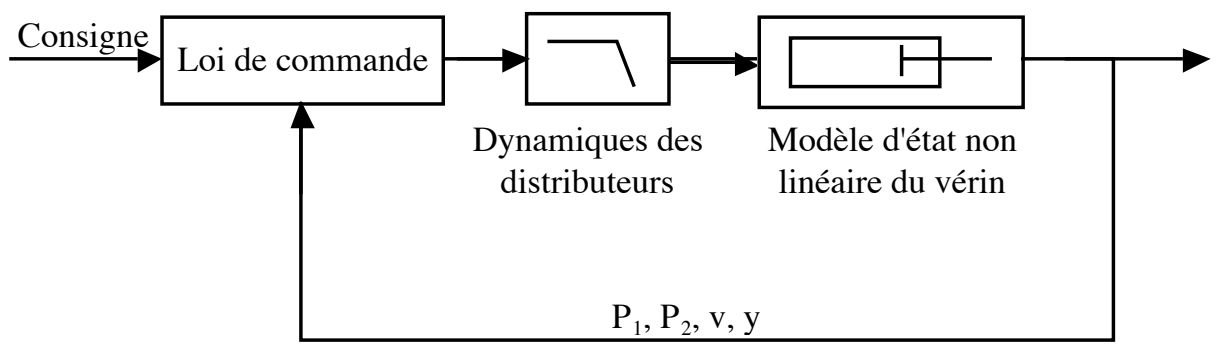

Figure 2. Schéma de simulation.

Ces résultats sont obtenus selon plusieurs valeurs de dynamiques des distributeurs. L'objectif est d'effectuer une comparaison des performances des systèmes bouclés obtenus, respectivement à partir de la loi de commande donnée dans ce papier et certaines lois de commande (désignées par commande II) proposées dans la littérature (Richard et al., 1997).

Dans le cadre de ces simulations, nous avons considéré un actionneur électrohydraulique constitué par un vérin symétrique double effet commandé par deux distributeurs identiques. Les paramètres du vérin et de la source hydraulique sont donnés dans le tableau 1. Le schéma de simulation est représenté par la figure 2.

\begin{tabular}{|l|l|l|}
\hline \multicolumn{1}{|c|}{ Paramètres du système } & \multicolumn{1}{c|}{ Valeur } & \multicolumn{1}{c|}{ Unité } \\
\hline $\mathrm{M}:$ masse & 500 & $\mathrm{Kg}$ \\
\hline $\mathrm{S}_{1}=\mathrm{S}_{2}:$ section utile & $9,456210^{-4}$ & $\mathrm{~m}^{2}$ \\
\hline$\ell:$ course du vérin & 0,2 & $\mathrm{~m}$ \\
\hline $\mathrm{P}_{\mathrm{s}}:$ pression source & $21010^{5}$ & $\mathrm{~Pa}$ \\
\hline $\mathrm{P}_{\mathrm{r}}:$ pression retour & $110^{5}$ & $\mathrm{~Pa}$ \\
\hline$\beta_{\mathrm{e}}:$ module de compressibilité effective & $1,410^{9}$ & $\mathrm{~Pa}$ \\
\hline
\end{tabular}

Tableau 1. Paramètres du système.

\begin{tabular}{|l|c|c|}
\hline & Position initiale (m) & Position finale (m) \\
\hline Simulation 1 & 0 & $-0,19$ \\
\hline Simulation 2 & $-0,05$ & 0 \\
\hline Simulation 3 & $-0,1$ & 0,1 \\
\hline
\end{tabular}

Tableau 2. Paramètres de simulation. 
Comme il a été précisé précédemment, la dynamique d'un distributeur est supposée être décrite par un système linéaire du premier ordre caractérisé par sa constante de temps $\tau$. Plusieurs valeurs de $\tau$ ont été considérées pour étudier l'influence de ce paramètre sur le comportement du processus électrohydraulique en boucle fermée. Par ailleurs, il convient de préciser, et vu les caractéristiques considérées, que la pulsation propre du vérin est $\omega_{0}=219,4 \mathrm{rd} / \mathrm{s}$.

Les figures (voir figures 3 - figures 5) donnent l'évolution des variables $\mathrm{P}_{1}, \mathrm{P}_{2}, \mathrm{v}$ et y pour différentes conditions initiales et finales (voir tableau 2) et ceci en faisant varier la constante de temps $\tau$. Plus précisément, les réponses des systèmes commandés sont tracées respectivement par un trait continu $(-)$ pour la commande I, par un trait discontinu régulier (------) pour la commande II et pour $\tau=0,45 \mathrm{~ms}$ et par un trait pointillé (……) pour la commande II et pour $\tau=45 \mathrm{~ms}$. Le tableau 3 donne les performances temporelles pour la simulation numéro 3 sur la variable position y où ts représente le temps de réponse à $2 \%$, à $5 \%$ et à $10 \%$.

\begin{tabular}{|c|c|c|c|c|c|}
\hline & $\tau[\mathrm{m}$ & $\rightarrow$ & 0,45 & 4,5 & 45 \\
\hline \multirow{3}{*}{ Loi de commande I } & \multirow{3}{*}{$\mathrm{t}_{\mathrm{s}}[\mathrm{s}]$} & $2 \%$ & 0,2248 & 0,2248 & 0,2248 \\
\hline & & $5 \%$ & 0,2134 & 0,2134 & 0,2134 \\
\hline & & $10 \%$ & 0,2024 & 0,2024 & 0,2024 \\
\hline \multirow{3}{*}{ Loi de commande II } & \multirow{3}{*}{$\mathrm{t}_{\mathrm{s}}[\mathrm{s}]$} & $2 \%$ & 0,1820 & 0,1920 & 0,3780 \\
\hline & & $5 \%$ & 0,1720 & 0,1780 & 0,3150 \\
\hline & & $10 \%$ & 0,1610 & 0,1660 & 0,2670 \\
\hline
\end{tabular}

Tableau 3. Performances temporelles.

Les résultats de simulation montrent que les performances de la loi de commande proposée dans ce papier sont tout à fait satisfaisantes. Par ailleurs, les réponses en boucle fermée (voir tableau 3) ne sont pas influencées par la dynamique des distributeurs. Par contre, on peut constater que les lois de commande données dans (Richard et al., 1997) fondées sur l'hypothèse que les dynamiques des distributeurs sont suffisamment rapides par rapport à celles de l'actionneur, sont sensibles aux dynamiques des distributeurs. En effet, les performances de ces lois de commande peuvent êtres détériorées, de manière significative, lors de dynamiques moins rapides des distributeurs.

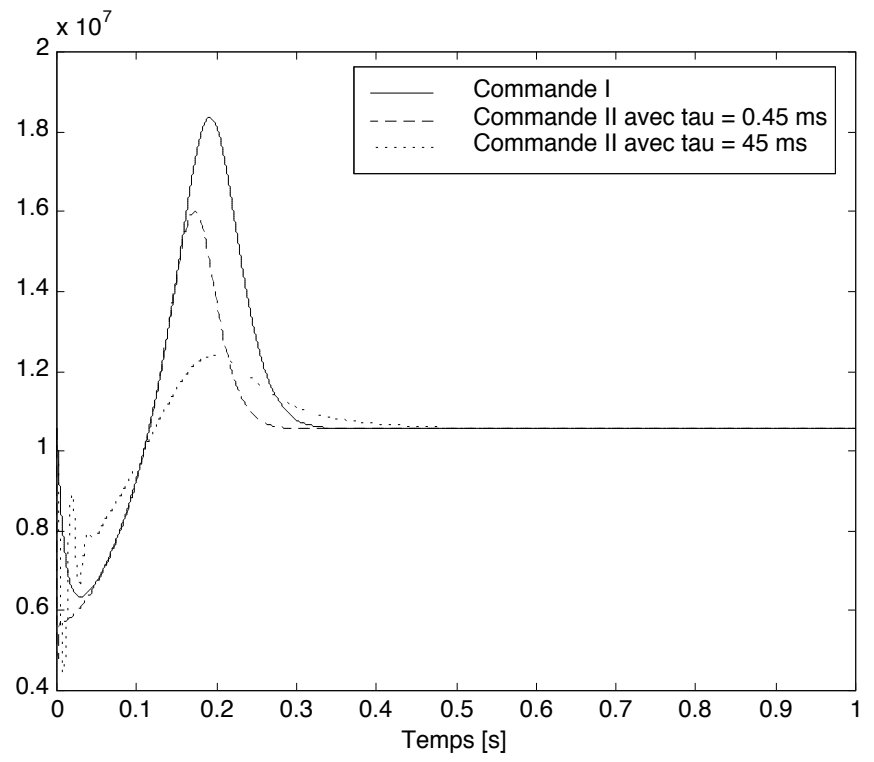

Figure 3-a. Pression $P_{1}[\mathrm{~Pa}]$ ( simulation 1). 


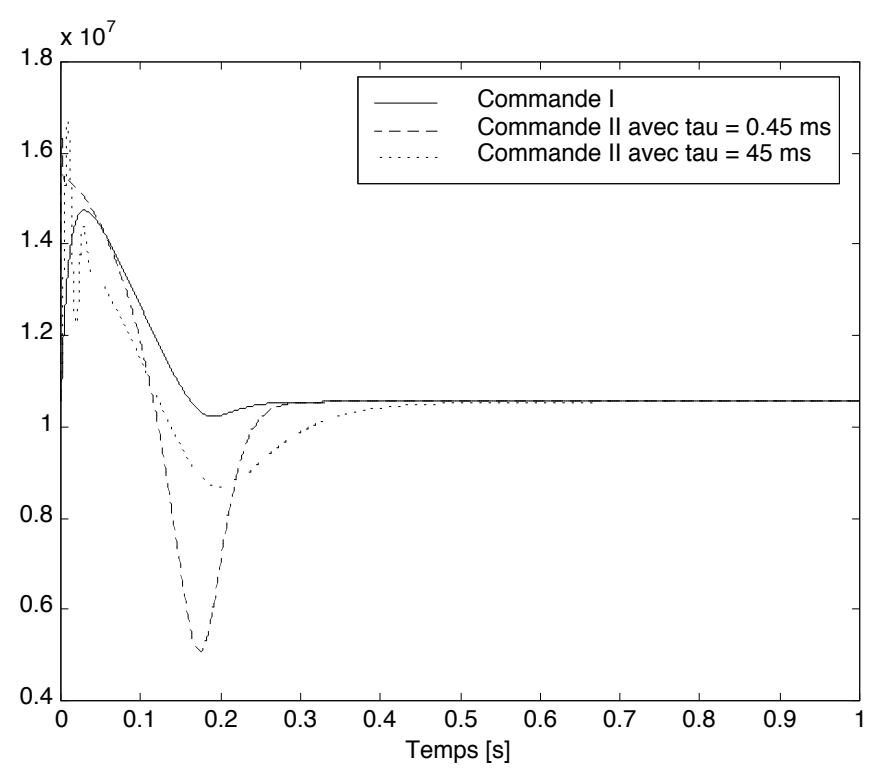

Figure 3-b. Pression $P_{2}[\mathrm{~Pa}]$ (simulation 1).

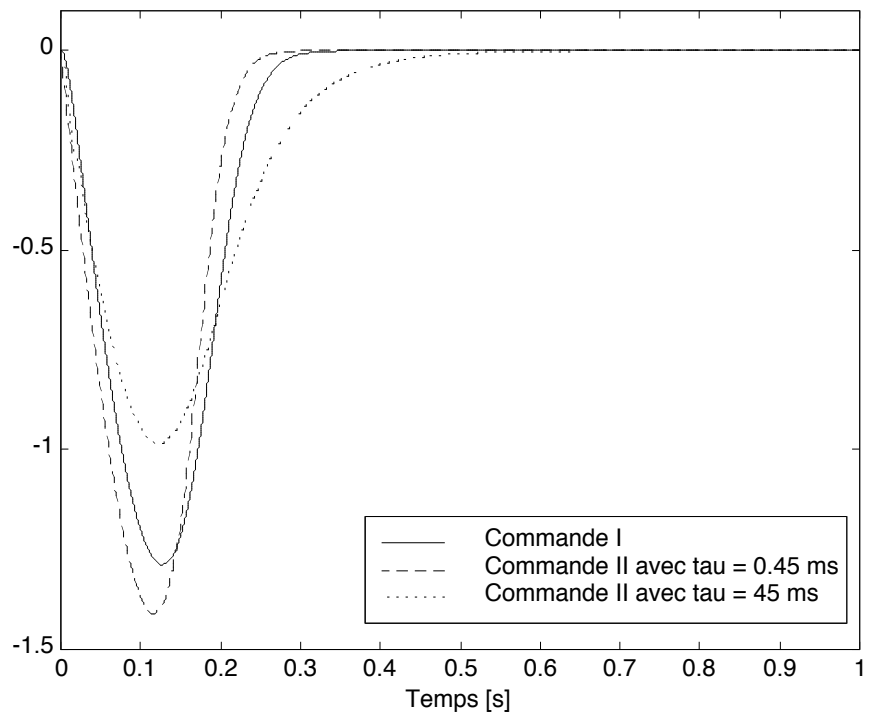

Figure 3-c. Vitesse $\left[m \cdot s^{-1}\right]$ (simulation 1). 


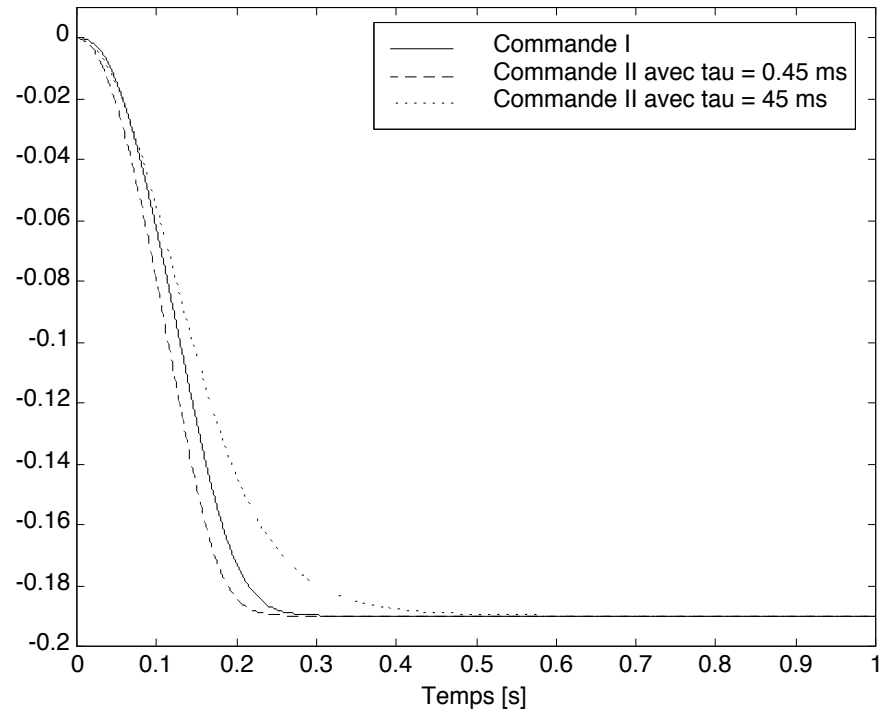

Figure 3-d. Position [m] (simulation 1).

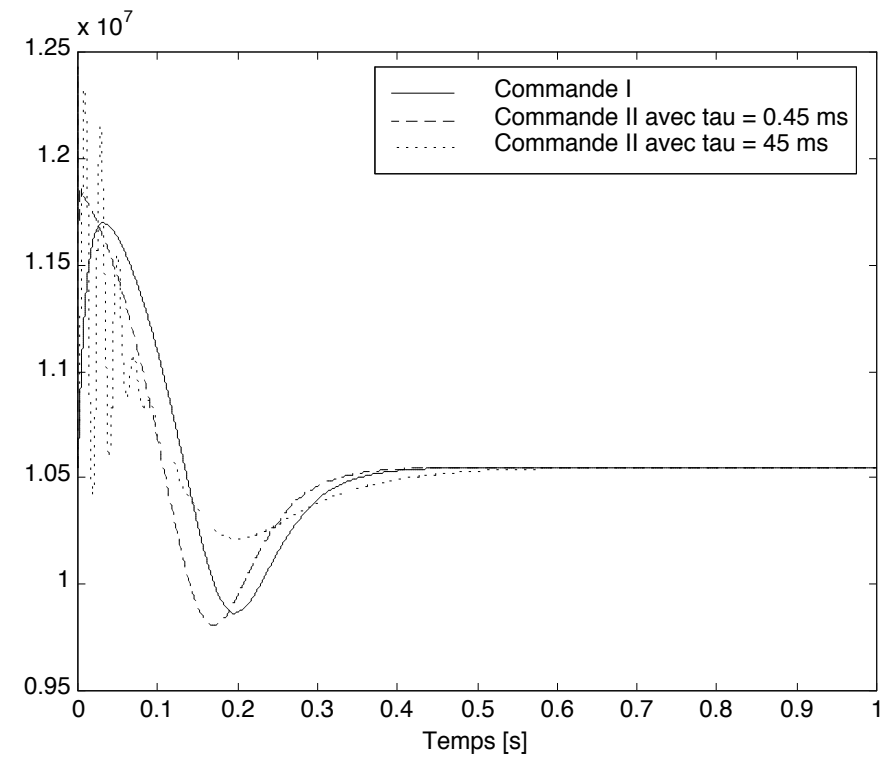

Figure 4-a. Pression $P_{1}[\mathrm{~Pa}]$ (simulation 2). 


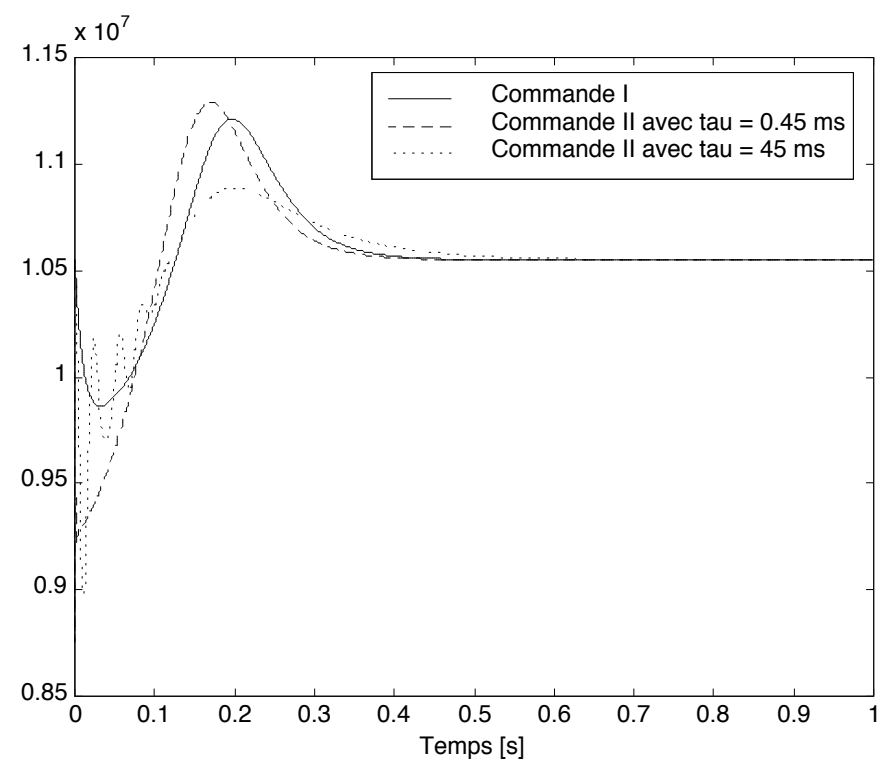

Figure 4-b. Pression $P_{2}[\mathrm{~Pa}]$ (simulation 2).

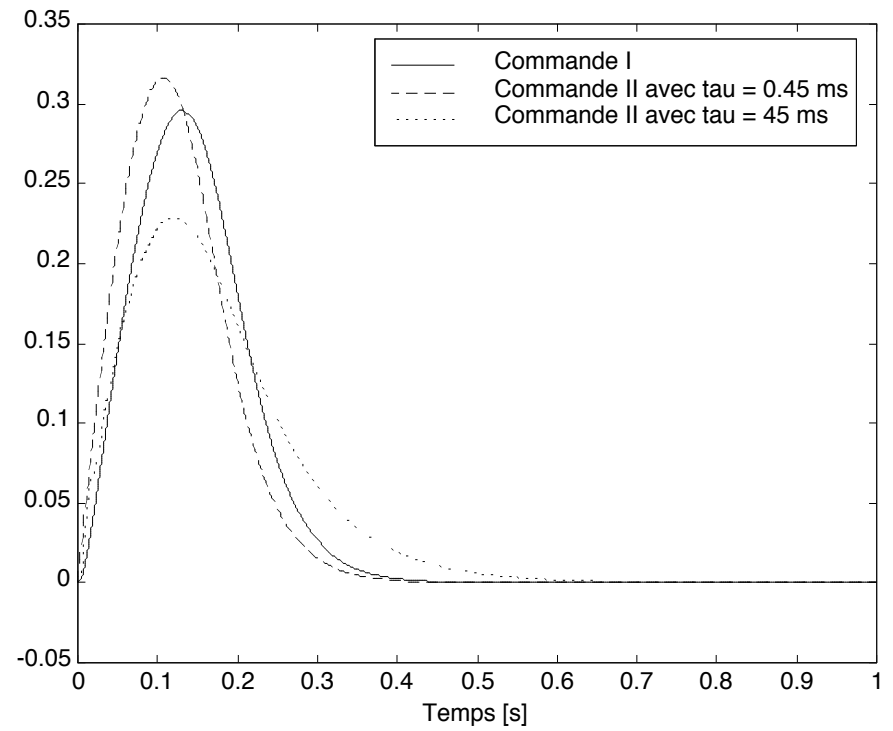

Figure 4-c. Vitesse $\left[m \cdot s^{-1}\right]$ (simulation 2). 


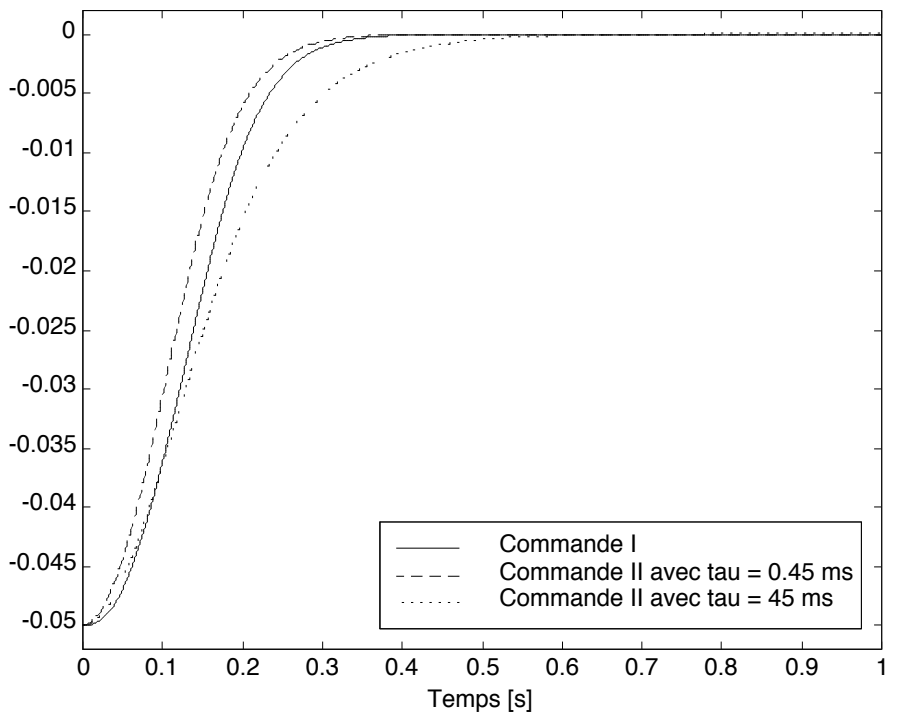

Figure 4-d. Position [m] (simulation 2).

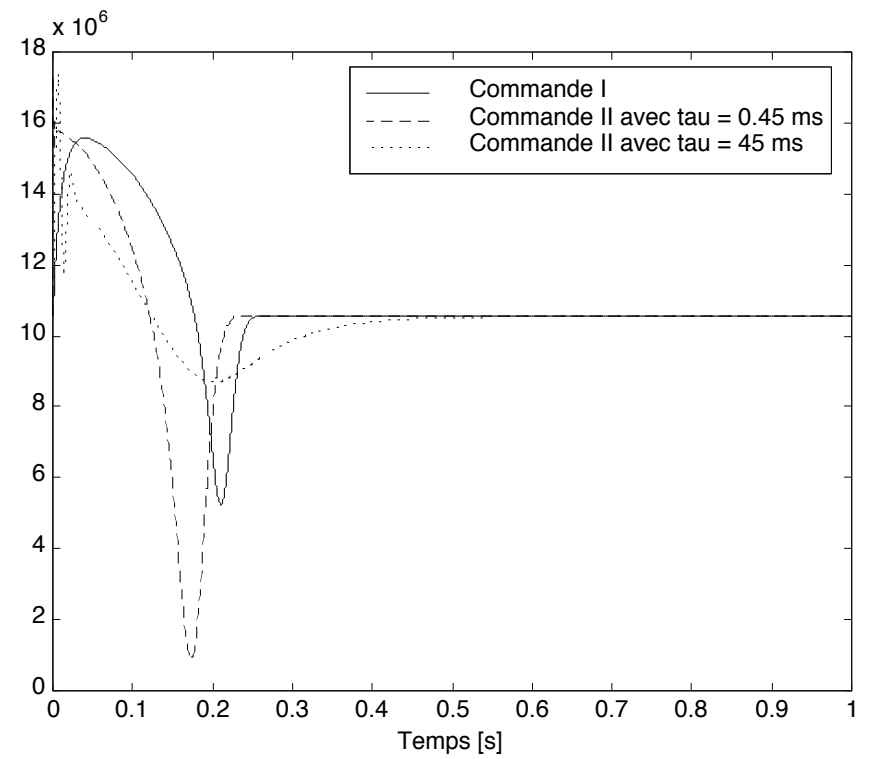

Figure 5-a. Pression $P_{1}[\mathrm{~Pa}]$ (simulation 3). 


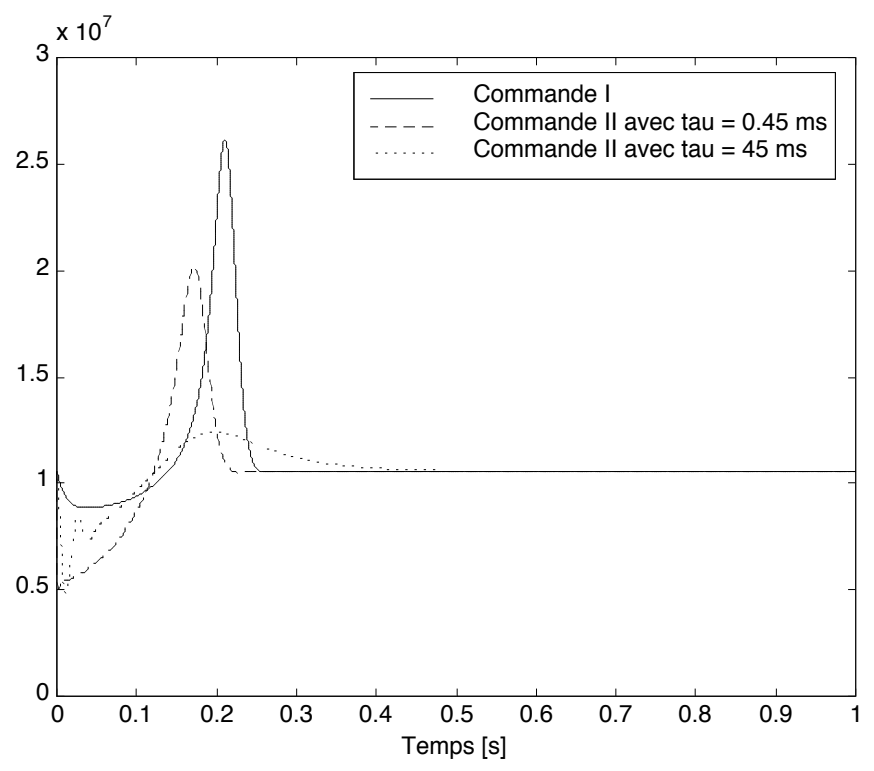

Figure 5-b. Pression $P_{2}[\mathrm{~Pa}]$ (simulation 3).

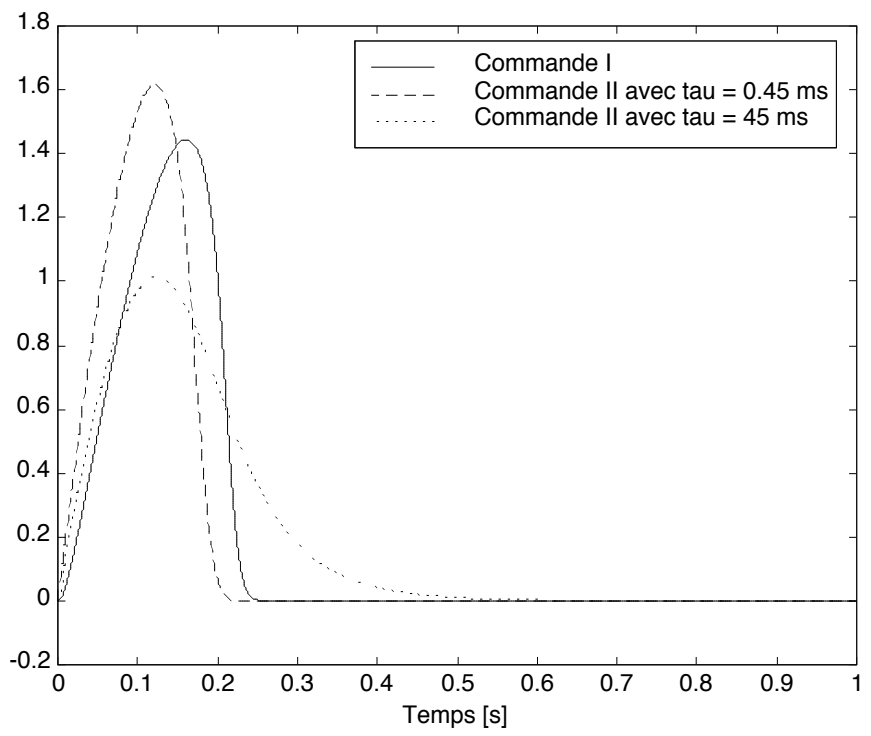

Figure 5-c. Vitesse $\left[m_{\cdot}^{-1}\right]$ (simulation 3). 


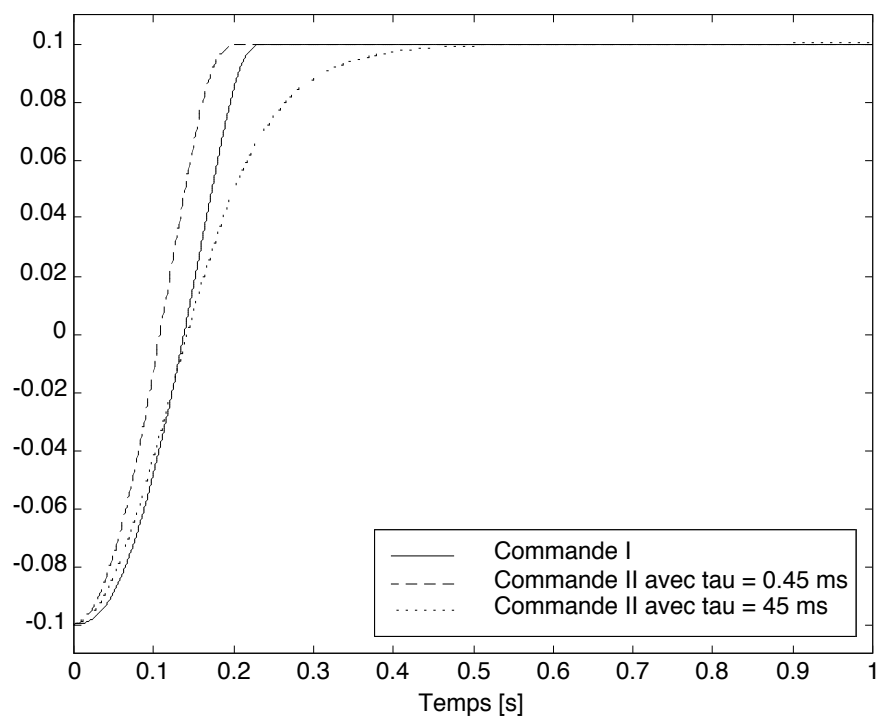

Figure 5-d. Position [m] (simulation 3).

\section{Conclusion}

Dans ce papier, nous avons présenté un nouveau résultat sur la stabilisation des systèmes non linéaires qui sont seulement continus (c'est-à-dire que les champs de vecteurs définissant les systèmes sont non dérivables). Nous avons ensuite appliqué ces résultats à la stabilisation d'actionneurs électrohydrauliques en tenant compte de la dynamique des distributeurs utilisés et ceci sans effectuer aucune linéarisation du modèle. Les simulations numériques montrent de meilleures performances de notre approche en comparaison avec une autre méthode non linéaire proposée dans la littérature.

La stratégie de stabilisation des processus électrohydrauliques, prenant en considération les dynamiques des distributeurs, présentée dans cet article a été donnée dans un cas ne tenant pas compte de toutes les contraintes d'un processus électrohydraulique. Néanmoins, et modulo de simples adaptations, cette stratégie peut être aisément étendue à certaines situations plus générales. On peut tenir compte par exemple, des frottements visqueux, des forces exogènes ou des fuites diverses.

Des travaux sont en cours pour étudier le cas où les dynamiques des distributeurs sont modélisées par une équation différentielle linéaire du second ordre. L'intérêt est dû au fait qu'une telle modélisation est encore plus réaliste. Cependant, et dans le cas d'une démarche similaire à celle présentée dans cet article, l'approche pose un problème d'ordre théorique qui n'est pas encore résolu à notre connaissance. 


\section{Bibliographie}

Blackburn J. F., Reethof, Shearer J. L., Mécanismes et servomécanismes à fluide sous-pression, tome 1, Paris, Dunod, 1966.

Byrnes C. I., Isidori A., « New results and examples in nonlinear feedback stabilization », Systems and Control Letters, vol. 12,1989 , p. 437-442.

Cloy Mc., Martin H. K., Control of fluid power analysis and design, New York, Jonh Wiley \& Sons, 1980.

Coron J.-M., Praly L., « Adding an integrator for the stabilization problem », Systems and Control Letters, vol. 17, 1991, p. 89-104.

Freeman R. A., Kokotovic P. V., Robust Nonlinear Control Design, Basel, Birkhauser, 1996.

Hahn H., Piepenbrink A., Leimbach K. D.., «Input/Output linearization of an electro servo-hydraulic actuator », Third IEEE Conference On Control Applications, Glasgow, 1994.

Khorrami F., Jain S., «Drive flexibility and friction in control of hydraulic actuators : a robust adaptive nonlinear control design », IEEE International Conference On Control Applications, Trieste, Italy, 1998.

Kokotovic P. V., Sussmann H., «A positive real condition for global stabilization of nonlinear systems », Systems and Control Letters, vol. 13, 1989, p. 125-133.

La Salle J. P., Lefschetz S., Stability by Liapunov's direct method with applications, New York, Academic Press, 1961.

Lebrun M., Contribution à une aide à l'analyse dynamique et à la conception de systèmes électrohydrauliques, Thèse d'état, Université Claude Bernard, Lyon, 1986.

Maré J.-C., Contribution à la modélisation, la simulation, l'identification et la commande d'actionneurs électrohydrauliques, Thèse d'état, Université Claude Bernard, Lyon, 1993.

Merritt H. E., Hydraulic control systems, New York, Jonh Wiley \& Sons, 1967.

Outbib R., Aggoune W., « Feedback stabilization of continuous systems by adding an integrator », International Journal of Applied Mathematics and Computer Science, vol. 9, n ${ }^{\circ}, 1999$, p. 71-88.

Outbib R., Jghima G., «Comments on the stabilization of nonlinear systems by adding an integrator », IEEE Transactions on Automatic Control, vol. 41, N${ }^{\circ} 12,1998$, p. 1804-1807.

Outbib R., Sallet G., « A reduction principle for the stabilization of nonlinear systems », Kybernetika, vol. 34, N5, 1994, p. 595-607.

Piché R., Pohjolainen S., Virvalo T., « Design of robust controllers for position servos using H-infinity theory », Proceding of Instr. Mech. Engineering, vol. 205, ํ14, 1992, p. 299-306.

Richard E., Outbib R., «Feedback stabilization of an electrohydraulic system », European Control Conference, Rome, Italy, 1995.

Richard E., Outbib R., Thomasset D., «Stabilisation d'un système électrohydraulique par retour d'état régulier », Journal Européen des Systèmes Automatisés, vol. 31, №7, 1997, p. 1173-1195.

Tanner H. G., Kyriakopoulos K. J., «Backstepping for non smooth systems», Automatica, N³9, 2003, p. 1259-1265.

Watton J., Fluid power systems, London, Prentice Hall, 1989.

Yao B., Bu F., Reedy J., Chiu G. T.-C., «Adaptive robust control of single-rod hydraulic actuators : theory and experiments », IEEE/ASME Transactions on Mechatronics, vol. 5, №1, 2000, p. 79-91. 